\title{
$\mathrm{CdH}_{2}$ 范德华分子的 CASSCF/SOCI 研究 \\ 李西平 \\ （昆明工学院基础部, 昆明 650093) \\ 马忠新戴树珊 \\ (云南大学化学系,屁明 650091)
}

\section{关勧调范德华分子、 $\mathrm{CdH}_{2} 、 \mathrm{CASSCF} / \mathrm{SOCI}$}

现代动力学的一个重要目的是在态-态水平上描述化学反应和能墨转移过程. 近几年发 展起来的半碰撞实验技术, 是在全属原子和试剂形成基态范德华分子的基础上,用选择性激光 激发金属原子至各电子态, 进而进行态一态水平上各种化学反应的研究. 虽然 $\mathrm{Cd}$ 原子与 $\mathrm{H}_{2}$ 的 全碰撞研究有不少报道, 但其半碰潼研究还未见报道. 本文对 $\mathrm{CdH}_{2}$ 范德华分子进行计算, 以 便对其半碰撞过程作出预测。

由于 $\mathrm{Cd}(z=48)$ 电子数较多, 相对论效应及电子相关效应都较为显著, 对 $\mathrm{Cd}-\mathrm{H}_{2}$ 体系 进行精确的从头算研究比较困难. 本文应用相对论碃势 (AREP) 全有效空间多组态自洽 (CASSCF) ab initio 和二级电子相关 (SOCI) 方法计算和分析了 $\mathrm{CdH}_{2}$ 体系的势能曲线和 电子结构, 并计算出各个态之间的跃迁能.

\section{一、计 算 方 法}

为了考虑 $\mathrm{Cd}$ 原子内层电子的㥵对论效应和降低计算量, 对 $\mathrm{Cd}$ 原子使用了 Christiansen 等的平均相对论有攽势 (AREP) 和相应的基组 ${ }^{\omega}$, 并将其基组分裂为双 5 . 对 $\mathrm{H}$ 原子则选自

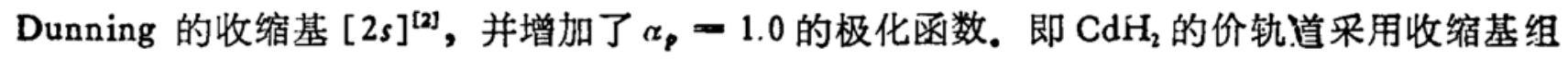
[2s $2 p 2 d / 2 s 1 p]$.

对 $\mathrm{Cd}+\mathrm{H}_{2}$ 的反应体系分别有 $\mathrm{Cd}$ 原子从侧面进攻 $\mathrm{H}-\mathrm{H}$ 键 $\left(C_{2 v}\right)$ 和端 位 进 攻 $\mathrm{H}_{2}$ 分 子 $\left(C_{\infty v 0}\right.$ ), 对应于范德华分子有相应的两种构型(即 $C_{2 v}$ 和 $C_{\infty v}$ ). 从计算技巧考虑, $C_{\infty}$ 型的计 算仍采用 $C_{2 v}$ 点群,分子置于 $y z$ 平面上.

$\mathrm{CdH}_{2}$ 作为 14 电子体系进行计算. 在 CASSCF 方法中, $\mathrm{Cd}$ 的 2 个电子 $(5 \mathrm{~s})$ 和 $\mathrm{H}_{2}$ 的两 个电子 $\left(\sigma_{1 s}\right)$ 作为有效电子, 以所有的方式分布在由 $\mathrm{Cd}$ 的 $5 s 、 5 p$ 和 $\mathrm{H}_{2}$ 的 $\sigma_{1 s} 、 \sigma_{1 s}^{*}$ 轨道构成的 有效空间中. CASSCF 组态数为 37 , 以 HF 函数为初始值进行迭代.

SOCI 计算以 CASSCF 优化出来的轨道为基础, 由于 Cd 原子的外实 (4d $\left.{ }^{10}\right)$ 电子的 CI 所 产生的组态空间过于巨大, 故在进行 SOCI 计算时, 将其冻结.

在优化出的平衡键长下, 从 $\mathrm{Cd}-\mathrm{H}_{2}$ 各态与基态能量之差, 以及各态之间的能量差求出䀓 迁能.

所有计算均由 HAMS 程序完成.

\section{二、结果和讨论}

1. $\mathrm{CdH}_{2}$ 范德华分子的构型 $\mathrm{CdH}_{2}$ 两种几何构型 $\left(C_{2 v}\right.$ 和 $\left.C_{\infty v 0}\right)$ 基态范德华分子的 键

本文 1990 年 10 月 15 日收到. 
长优化值列于表 1 , 此值均为 $\mathrm{Cd}$ 原子至氢分子键中心的距离. 表中值是对 $\mathrm{CdH}_{2}$ 范德华分子 的构型作出的预测. 可见, $\mathrm{Cd}$ 以两种方式进攻 $\mathrm{H}_{2}$ 都可能形成范德华分子. 不同的是 $C_{\infty}$ 构 型的分子相对稳定一些,并且 $\mathrm{H}_{2}$ 分子中心与 $\mathrm{Cd}$ 原子之间的距离略近一些.

2. $\mathrm{CdH}_{2}$ 范德华分子的电子梞 表 2 是 $\mathrm{CdH}_{2}$ 范德华分子的电子态. 图 1 是用 $\mathrm{SOCI}$ 方法得出的两种构型的范德华分子各电子态的势能曲线.

表 $1 \mathrm{CdH}_{2}$ 范德华分子键长 $(\AA)$

\begin{tabular}{c|c|c}
\hline 方 法 & $R\left(C_{20}\right)$ & $R\left(C_{\ldots o}\right)$ \\
\hline SCF & 6.12 & 6.00 \\
\hline CASSCF & 6.01 & 5.59 \\
\hline SOCI & 5.17 & 4.96 \\
\hline
\end{tabular}

表 $2 \mathrm{CdH}_{2}$ 范德华分子的电子态

\begin{tabular}{|c|c|c|c|}
\hline \multirow{2}{*}{ 轨道占据 } & \multirow{2}{*}{ 电子自旋 } & \multicolumn{2}{|c|}{ 分 子 态 } \\
\hline & & $c_{20}$ & $c_{\infty}$ \\
\hline$S_{s}^{2}(\mathrm{Cd})+\sigma_{i s}^{2}(\mathrm{H})$ & 低自旋 & $\mathbb{I}^{2} A_{1}$ & $1^{1} \Sigma^{+}$ \\
\hline \multirow{2}{*}{$5 s^{1}, 5 p_{z}^{1}(\mathrm{Cd})+\sigma_{1 s}^{2}(\mathrm{H})$} & 低自掟 & $2^{1} A_{t}$ & $2^{1} \Sigma^{+}$ \\
\hline & 高自旋 & ${ }^{3} A_{1}$ & ${ }^{s} \Sigma^{+}$ \\
\hline $5 s^{1}, 5 p \mathrm{i}(\mathrm{Cd})+\sigma_{1}^{2}(\mathrm{H})$ & \multirow{2}{*}{ 低 自 旅 } & ${ }^{1} B_{1}$ & \multirow{2}{*}{ ' $I$} \\
\hline $5 s^{1}, 5 p_{y}^{\prime}(\mathrm{Cd})+\sigma_{i s}^{2}(\mathrm{H})$ & & $\mathbf{B}_{2}$ & \\
\hline $5 s^{2}, 5 p_{n}^{1}(\mathrm{Cd})+\sigma_{1 s}^{2}(\mathrm{H})$ & \multirow{2}{*}{ 高自旋 } & $\mathrm{B}_{1}$ & \multirow{2}{*}{${ }^{3} \pi$} \\
\hline $5 s^{1}, 5 p_{y}^{1}(\mathrm{Cd})+\sigma_{i s}^{2}(\mathrm{H})$ & & $3^{3} B_{2}$ & \\
\hline
\end{tabular}

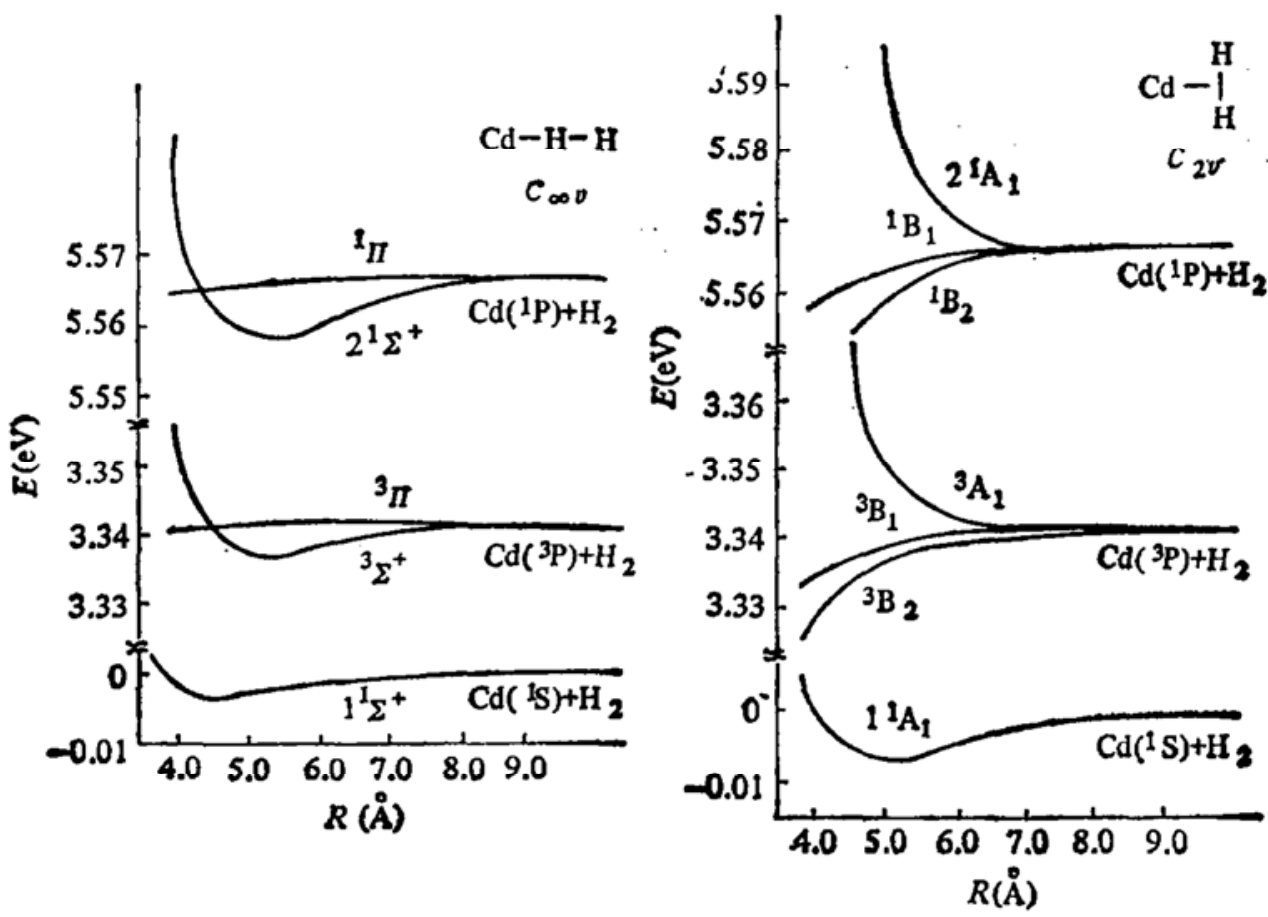

图 $1 \mathrm{CdH}_{2}$ 范德华分子电子态的势能曲线 
从图中可以看出, 在 $C_{20}$ 构型中, 基态 $1^{1} A_{1}$ 是束缚态, 在 $5.174 \AA$ 处出现最低点, $\mathrm{CdH}_{2}$ 能 相对稳定存在, 形成范德华分子. $2^{1} \mathrm{~A}_{1}$ 和 ${ }^{3} \mathrm{~A}_{1}$ 态是排斥态, 这两个态都是一对反自旋电子排 布 在 $\sigma_{1 s}\left(\mathrm{H}_{2}\right)$ 成键轨道中, 一个电子在 $5 s(\mathrm{Cd})$ 轨道, 另一个电子以反自旋或同自旋排布在 $5 \mathrm{ps}$ (Cd) 轨道中而形成两个不同的态. 由于 $5 p_{x}(\mathrm{Cd})$ 轨道比 $5 s(\mathrm{Cd})$ 轨道较为伸展, 排斥作用增 强, 形成排斥态.

${ }^{1} \mathrm{~B}_{1} 、{ }^{1} \mathrm{~B}_{2} 、{ }^{3} \mathrm{~B}_{1} 、{ }^{3} \mathrm{~B}_{2}$ 都是吸引态. 这几个态均有一个电子在 $5 s(\mathrm{Cd})$ 轨道, 而另一个电子处于 $5 p_{y}(\mathrm{Cd})$ 或 $5 p_{x}(\mathrm{Cd})$ 轨道, 与 $\sigma_{18}\left(\mathrm{H}_{2}\right)$ 轨道中的两个电子排斥较小. 可以推测, 随着 $R$ 的减小, 将可导致如下反应:

$$
\mathrm{Cd}(5 s 5 p)+\mathrm{H}_{2} \longrightarrow \mathrm{CdH}+\mathrm{H},
$$

其中 ${ }^{1} \mathrm{~B}_{2}$ 和 ${ }^{3} \mathrm{~B}_{2}$ 随着 $R$ 的减小能量降低较快, 反应更容易发生. 这是因为被激发的 $\mathrm{Cd}$ 原子的 单占据 $p_{y}$ 轨道能够与 $\mathrm{H}_{2}$ 分子的 $\sigma_{1 s}^{*}\left(\mathrm{H}_{2}\right)$ 轨道很好地重叠. 此外, 氢分子全充满的 $\sigma_{16}$ 轨道也能 和金属原子半充满的 $s$ 轨道发生交互作用。

$C_{\infty}$ 构型中,除基态 $1^{1} \Sigma^{+}$在 $4.96 \AA$ 处出现最低点外, $2^{1} \Sigma^{+}$和 ${ }^{3} \Sigma^{+}$态在 $5.721 \AA$ 和 $5.579 \AA$ 也分别出现能量最低点. 从轨道对称性和电子结构分析可知, 这是由于 $\sigma_{1 /}^{*}\left(\mathrm{H}_{2}\right)$ 空轨道与半充 满的 $5 s(\mathrm{Cd})$ 和 $5 p_{s}(\mathrm{Cd})$ 轨道能较有效地重叠.

与 $C_{2}$ 构型下 ${ }^{1} \mathrm{~B}_{1} 、{ }^{3} \mathrm{~B}_{1} 、{ }^{1} \mathrm{~B}_{2}$ 和 ${ }^{3} \mathrm{~B}_{2}$ 态表现为吸引的势能曲线迥然不同, ${ }^{1} \Pi$ 和 ${ }^{3} \Pi$ 态的势能曲 线随着 $R$ 的减小仅稍有下降, 这是因为 $p_{s}(\mathrm{Cd})$ 或 $p_{y}(\mathrm{Cd})$ 轨道中的电子与 $\sigma_{1 s}^{*}\left(\mathrm{H}_{2}\right)$ 空轨道不 能有效地重叠. 与 $C_{20}$ 松型相比, 可推测, 从端位进攻发生反应生成 $\mathrm{CdH}$ 产物较为困难, 这与 文献资料中对 $\mathrm{MgH}_{2}{ }^{[3]}$ 和 $\mathrm{HgH}_{2}{ }^{[0]}$ 等体系的研究结果相一致.

表 $3 \mathrm{CdH}_{2}$ 电子态的跃迁能 $\Delta E_{t}\left(\mathrm{~cm}^{-1}\right)$

\begin{tabular}{|c|c|c|c|}
\hline \multicolumn{2}{|c|}{$C_{20}$} & \multicolumn{2}{|c|}{$c_{\ldots}$} \\
\hline 跃 迁 态 & $\Delta E$ & 昕 灭 态 & $\Delta E_{t}$ \\
\hline $\begin{aligned}{ }^{2} A_{1} & \\
& { }^{3} A_{1} \\
& B_{1} \\
{ }^{1} A_{1} \longrightarrow & { }^{1} B_{2} \\
& { }^{3} B_{1} \\
& \\
& { }^{3} B_{2}\end{aligned}$ & $\begin{array}{l}45045.07 \\
27009.73 \\
44882.08 \\
44851.60 \\
26936.81 \\
26925.38\end{array}$ & $\begin{aligned} 2^{1} \Sigma^{+} & { }^{3} \Sigma^{+} \\
1^{1} \Sigma^{+} \longrightarrow & { }^{\prime} \Pi \\
& \end{aligned}$ & $\begin{array}{l}44879.92 \\
26950.34 \\
44912.18 \\
26966.58\end{array}$ \\
\hline 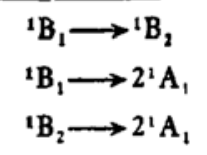 & $\begin{array}{r}30.48 \\
162.99 \\
193.47\end{array}$ & $\begin{array}{l}{ }^{1} \Pi \longrightarrow 2^{\prime} \Sigma^{+} \\
{ }^{\prime} \Sigma^{+} \longrightarrow{ }^{\prime} \Pi I\end{array}$ & $\begin{array}{l}32.20 \\
16.24\end{array}$ \\
\hline
\end{tabular}

3. $\mathrm{CdH}_{2}$ 电子态的跃迁能即迁能 $\left(\Delta E_{t}\right)$ 是在 $\mathrm{CdH}_{2}$ 范德华分子平衡构型下, 某态与 基态或与另一个态之间的能量差, 以此预测由范德华分子 $\mathrm{CdH}_{2}$ 的某态至其它态所唐之能量. 借助表 3 中数值, 按选择定则可以找到各容许跃迁态之 $\Delta E_{t}$, 其值可分辩. 这预示对于 $\mathrm{CdH}_{2}$ 体系可通过半碰撞技术, 由其范德华分子的基态激发至指定激发态, 以进行态-态反应的研究.

对 $\mathrm{CdH}_{2}$ 范德华分子的势能曲线及跃迁能等研究表明, 当基态 $\mathrm{Cd}$ 原子与氢分子相互作用 生成范德华分子之后, 激发电子使其处于 ${ }^{1} \mathrm{~B}_{1} 、{ }^{1} \mathrm{~B}_{2}$ 激发态以发生半碰撞, 这与激发态的 $\mathrm{Cd}\left({ }^{\mathrm{P}} \mathrm{P}\right)$ 与 $\mathrm{H}_{2}$ 的猝灭全碰撞相对应 ${ }^{\text {" }}$. 


\section{参考文蝴}

[1] Lajohn, P.A. et al., J. Chem. Phys., 87(1987),2821.

[2] Dunning, T.H., Hay, P.J., Modern Theoretical Chemistry 3(Ed. Schaefer III, H.F. ), Plenum Press. New York, 1977,1.

[3] Chaquin, P. et al., J. Phys. Chem., S9(1985),2813.

[4] Bernier, A., Millie, P., Chem. Phys. Lett., 134(1987), 245.

[5] Breckenridge, W.H. et al., J. Phys. Chem., 82(1978),1484. 